\title{
Stereoselective total synthesis of cochliomycin A
}

\author{
Linlin Wang ${ }^{\mathrm{a}}$, Yangguang Gao ${ }^{\mathrm{b}}$, Jun Liu ${ }^{\mathrm{a}, *}$, Chao Cai ${ }^{\mathrm{a}}$, Yuguo Du ${ }^{\mathrm{a}, \mathrm{b}, *}$ \\ a State Key Laboratory of Environmental Chemistry and Eco-Toxicology, Research Center for Eco-Environmental Sciences, Chinese Academy of Sciences, \\ Beijing 100085, China \\ ${ }^{\mathrm{b}}$ School of Chemistry and Chemical Engineering, University of Chinese Academy of Sciences, Beijing 100049, China
}

\section{A R T I C L E I N F O}

\section{Article history:}

Received 13 January 2014

Received in revised form 24 February 2014

Accepted 2 March 2014

Available online 6 March 2014

\section{Keywords:}

Cochliomycin A

Total synthesis

Resorcylic acid lactones

\begin{abstract}
A B S T R A C T
A convergent and stereoselective synthesis of cochliomycin A, a 14-membered resorcyclic acid lactone, based on chiron approach is described. The key reactions involved olefin cross-metathesis and sodium hydride promoted one-pot intramolecular lactonization. L-Arabinose was used as a chiral pool material for the construction of the key fragment.
\end{abstract}

(c) 2014 Elsevier Ltd. All rights reserved.

\section{Introduction}

In recent years, resorcylic acid lactones (RALs) have gained considerable attention, as many of them possess remarkable and diverse biological activities. ${ }^{1}$ Cochliomycin A-C (1-3, Fig. 1), three

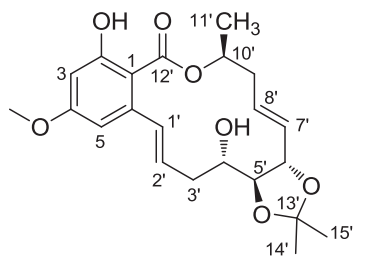

Cochliomycin A (1)<smiles>COc1cc(O)c(C(=O)O[C@@H](C)CCC[C@@H](O)[C@H](O)[C@H](O)C/C=C/c2c(Cl)c(OC)cc(OC)c2Cl)c(O)c1</smiles>

Cochliomycin C (3)<smiles>COc1cc(O)c(C(=O)O[C@H](C)C/C=C/[C@H](O)[C@H]2OC(C)(C)O[C@@H]2C/C=C/c2cc(OC)cc(O)c2C)c(O)c1</smiles>

Cochliomycin B (2)

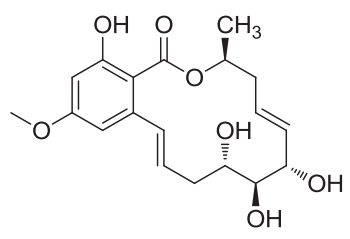

Zeaenol (4)
Fig. 1. Structures of cochliomycin $\mathrm{A}-\mathrm{C}(\mathbf{1}-\mathbf{3})$ and zeaenol (4).

\footnotetext{
* Corresponding authors. E-mail addresses: junliu@rcees.ac.cn (J. Liu), duyuguo@ rcees.ac.cn (Y. Du).
}

new 14-membered resorcyclic acid lactones, together with the known zeaenol (4), were isolated from the culture broth of Cochliobolus lunatus in 2011. ${ }^{2}$

The cochliomycin A (1) and B (2) comprised similar 14membered resorcyclic acid lactone skeleton with a rare natural acetonide group. The relative configurations of cochliomycin $A$ and $B$ were established on the basis of extensive spectroscopic analysis including 1D NOE and 2D NOESY experiments. The only difference between these two compounds is the acetonide group, which has never been found before in any natural resorcylic acid lactone. The absolute configurations of cochliomycin A and B were further confirmed by treatment of zeaenol (4) with 2,2-dimethoxypropane in the presence of $p$-toluenesulfonic acid (PTSA) resulted in a mixture of cochliomycin A and B. Cochliomycin A was found to show potent antifouling activity against the larval settlement of the barnacle Balanus amphitrite and moderate antibacterial activity against Staphylococcus aureus. It is noteworthy that the unusual acetonide moiety in cochliomycin A probably contributes to the antifouling activity because cochliomycin A showed better antifouling activity with $\mathrm{EC}_{50}$ value $(1.2 \mu \mathrm{g} / \mathrm{mL})$, approximately threefold over zeaenol (4) $(5.0 \mu \mathrm{g} / \mathrm{mL})$.

Due to its novel structure and potent biological activity, cochliomycin A constitutes an ideal target for total synthesis. Very recently, cochliomycin A was firstly synthesized by Nanda's group from $\mathrm{L}-(+)$-tartaric acid in $6.5 \%$ overall yields employing Keck allylation, Julia-Kocienski olefination and a late-stage RCM reaction as the key steps. ${ }^{3}$ As part of our interest in the total synthesis of various complex natural products based on carbohydrate skeletons, ${ }^{4}$ we herein report a convergent total synthesis of cochliomycin A using L-arabinose as the chiral pool material. 
Our retrosynthesis analysis of cochliomycin A is summarized in Scheme 1. To construct the 14-membered macrocyclic core, we planned to utilize macrolactonization of the precursor $\mathbf{5}$, which could be obtained by Stille coupling between the stannane $\mathbf{6}$ and the known triflate 7 . The key intermediate $\mathbf{6}$ could be obtained from the epoxide $\mathbf{8}$ via an easy reaction sequence. The trans olefin moiety at $C 7^{\prime} / C 8^{\prime}$ in 8 could be installed by olefin cross-metathesis of the epoxide $\mathbf{9}$ and the olefinic side chain $\mathbf{1 0}$, which could be accessible readily from L-arabinose and (S)-(-)-propylene oxide, respectively. This approach was based on the fact that the three desired continuous stereogenic centers in the cochliomycin A (1) could directly be mapped onto natural L-arabinose.
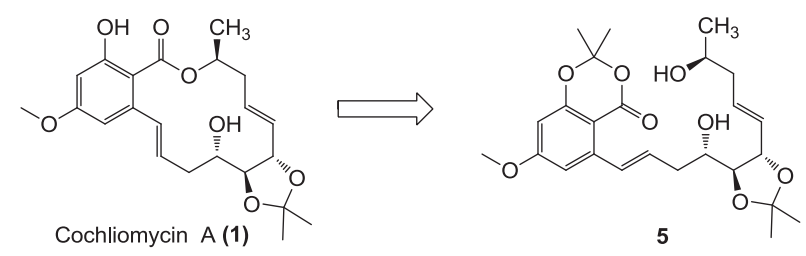
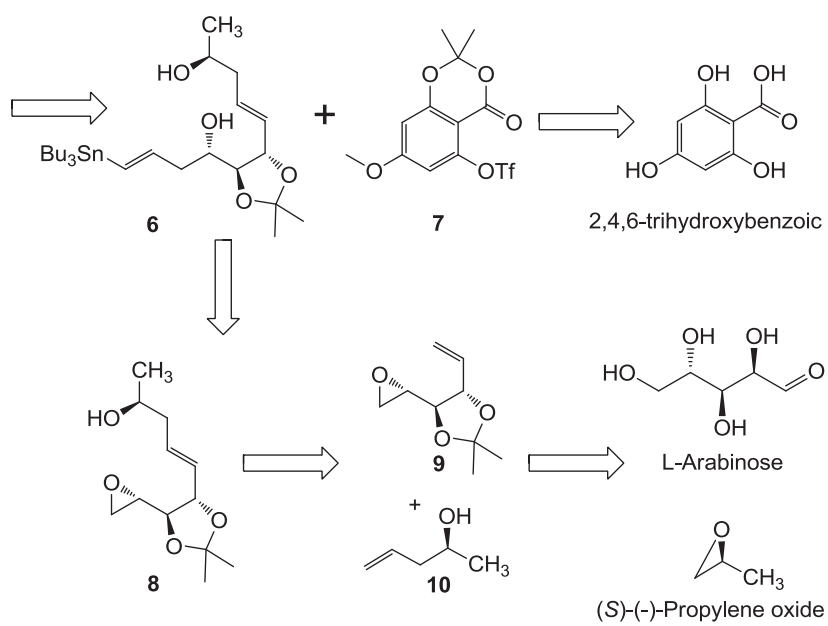

Scheme 1. Retrosynthetic analysis of cochliomycin A (1). 1,2-diol 13. Regioselective monotosylation ${ }^{8}$ of $\mathbf{1 3}$ followed by base treatment gave the epoxide $\mathbf{9}$ in 78\% yield for two steps. ${ }^{9}$

The known homoallylic alcohol $\mathbf{1 0}$ was prepared by treating commercially available (S)-(-)-propylene oxide with vinylmagnesium bromide in the presence of CuI. ${ }^{10}$ Exposure the mixture of the epoxide $\mathbf{9}$ and the homoallyl alcohol $\mathbf{1 0}$ in 1:1.5 ratio to Grubbs second generation catalyst in dry DCM under reflux provided the desired $(E)$-alkene in $85 \%$ yield along with small amounts of the dimer of $\mathbf{1 0} .^{11}$ The ring opening of the epoxide $\mathbf{8}$ with lithium acetylide from TMS-acetylene in the presence of $\mathrm{BF}_{3} \cdot \mathrm{Et}_{2} \mathrm{O}$ gave the corresponding homopropargylic alcohol 15 in 78\% yield. ${ }^{12}$ After deprotection of the TMS group in $\mathbf{1 5}$ with $\mathrm{K}_{2} \mathrm{CO}_{3}$ in methanol, the resulting terminal alkyne was treated with NBS ( $N$-bromosuccinimide) in the presence of silver nitrate to afford the 1-bromoalkyne 17 in almost quantitative yield. ${ }^{13}$ According to the Guibe's protocol, Pd-catalyzed hydrostannylation of the bromoalkyne $\mathbf{1 7}$ smoothly yielded the desired (E)-vinyl stannane $\mathbf{6}$ at room temperature (Scheme 3). ${ }^{14}$

Stille cross-coupling of $\mathbf{6}$ and the known aryl triflate $\mathbf{7}^{15}$ proceeded smoothly to give the alkene $\mathbf{5}$ in $81 \%$ yield. ${ }^{16}$ Hydrolysis of 5 with $\mathrm{LiOH}$ in a solution of $\mathrm{THF} / \mathrm{H}_{2} \mathrm{O}(2: 1)$ afforded an almost quantitative yield of the free acid $\mathbf{1 8}$ (Scheme 4).

However, difficulties were encountered during the attempted macrocyclization of the resulting carboxylic acid to the corresponding macrolide. The Yamaguchi macrolactonization strategy ${ }^{17}$ was first explored. Unfortunately, only a trace amount of cochliomycin A was obtained under this condition. Attempts to improve the yield by changing reaction concentration or going to higher temperature were fruitless. The Corey-Nicolaou double activation strategy ${ }^{18}$ was also examined and no desired product was observed. Because of the failure of the original strategy for constructing macrolide from free acid 18, a backup approach was adopted. Finally, according to De Brabander's conditions, ${ }^{19}$ one-pot intramolecular lactonization was achieved with excess NaH in dry DMF, leading to cochliomycin A (1) in reasonable yield (Scheme 4). All the spectroscopic data and specific rotation data of our synthetic cochliomycin $\mathrm{A}$ are in good agreement with those reported values for natural product $\left\{[\alpha]_{\mathrm{D}}^{24}+10.5(c 0.6, \mathrm{MeOH})\right.$; Ref. $3:[\alpha]_{\mathrm{D}}^{30}+10.6(c$ $0.5, \mathrm{MeOH})$; Ref. 2: $\left.[\alpha]_{\mathrm{D}}^{24}+10.5(c 0.43, \mathrm{MeOH})\right\}$.

\section{Conclusions}

In conclusion, a convergent and stereoselective synthesis of the resorcyclic acid lactone, cochliomycin $\mathrm{A}$, has been achieved starting from readily available chiral pool L-arabinose. The key reactions involved olefin cross-metathesis, Stille cross-coupling and sodium hydride promoted one-pot intramolecular lactonization. Further synthetic studies on other cochliomycins and their biological

evaluation are currently under investigation in our laboratory.
Accordingly, the synthesis of the key intermediate $\mathbf{9}$ started from L-arabinose and is outlined in Scheme 2. The known aldehyde 11 was prepared from L-arabinose in three steps according to the reported procedure. ${ }^{5}$ Wittig reaction ${ }^{6}$ of the aldehyde $\mathbf{1 1}$ with triphenylphosphonium methylidene followed by selective hydrolysis ${ }^{7}$ of the terminal acetonide with $75 \%$ aqueous acetic acid afforded the<smiles>CC1(C)OC([C@H]2COC(C)(C)O2)[C@@H](C=O)O1</smiles>

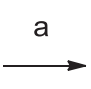<smiles>C=C[C@H]1OC(C)(C)O[C@H]1[C@H]1COC(C)(C)O1</smiles><smiles>C=C[C@H]1OC(C)(C)O[C@H]1[C@H](O)CO</smiles><smiles>C[As]=[Te]</smiles><smiles>C=C[C@H]1OC(C)(C)O[C@@H]1[C@H](O)CO</smiles>

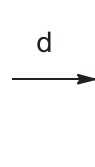


<smiles>C=CC[C@H](C)O</smiles><smiles>C#CC[C@H](O)[C@H]1OC(C)(C)O[C@@H]1/C=C/C[C@@H](C)O</smiles><smiles>C[C@H](O)C/C=C/[C@H]1OC(C)(C)O[C@H]1C1CO1</smiles><smiles>C[C@H](O)C/C=C/[C@H]1OC(C)(C)O[C@H]1[C@H](O)CC#CBr</smiles><smiles>CC(O)C/C=C\[C@H]1OC(C)(C)O[C@H]1[C@H](O)CC#CS(C)(C)C</smiles>

15<smiles>C[C@H](O)C/C=C/[C@H]1OC(C)(C)OC1[C@H](O)C/C=C/[Sn]C(C)(C)C</smiles>

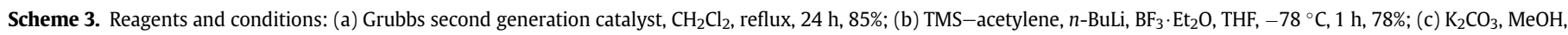
12 h, 86\%; (d) $\mathrm{NBS}, \mathrm{AgNO}_{3}$, acetone, 1 h, 97\%; (e) $\mathrm{PdCl}_{2}\left(\mathrm{PPh}_{3}\right)_{2}, \mathrm{Bu}_{3} \mathrm{SnH}$, THF, rt, $30 \mathrm{~min}, 85 \%$.

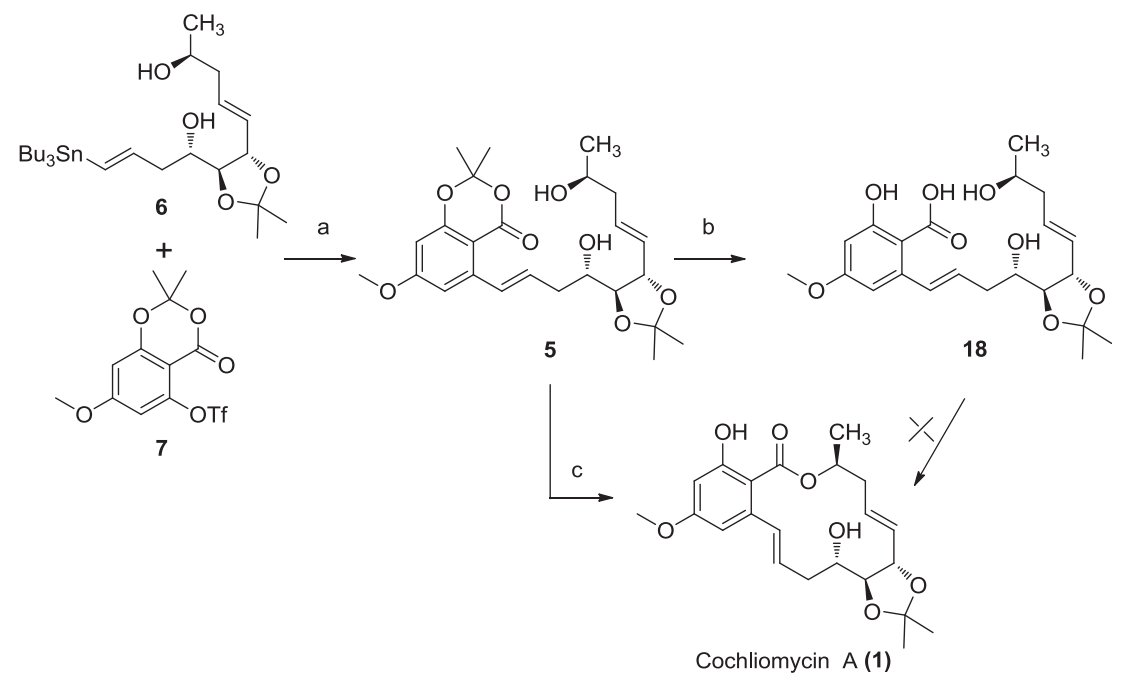

Scheme 4. Reagents and conditions: (a) Pd(dba) $)_{2} \mathrm{AsPh}_{3}, \mathrm{LiCl}, \mathrm{DMF}, 60{ }^{\circ} \mathrm{C}, 2 \mathrm{~h}, 81 \%$; (b) $\mathrm{LiOH} \cdot \mathrm{H}_{2} \mathrm{O}, \mathrm{THF} / \mathrm{H}_{2} \mathrm{O}(2: 1), 24 \mathrm{~h}$. (c) $\mathrm{NaH}, \mathrm{dry} \mathrm{DMF}, 0{ }^{\circ} \mathrm{C}$ to rt, 1 h, $46 \%$.

\section{Experimental section}

\subsection{General}

All anhydrous solvents were purified according to standard methods. All commercially available reagents were used without further purification. Reactions were monitored by thin-layer chromatography (TLC) on gel $\mathrm{F}_{254}$ plates. Flash chromatography (FC) was performed using silica gel (200-300 mesh) according to the standard protocol. Optical rotations were recorded in either a 5$\mathrm{cm}$ microcell or a $2.5-\mathrm{cm}$ microcell. High-resolution mass spectrometry data (HRMS) were acquired with a Q-TOF analyzer. ${ }^{1} \mathrm{H}$ and ${ }^{13} \mathrm{C}$ NMR spectra were recorded on a Bruker $400 \mathrm{MHz}$ (100 MHz for $\left.{ }^{13} \mathrm{C}\right)$ NMR spectrometer.

4.1.1. (4R,4'S,5S)-2,2,2',2'-Tetramethyl-5-vinyl-4, 4'-bi(1,3-dioxolane) (12). To a stirred suspension of methyl triphenylphosphonium bromide (131 g, $367 \mathrm{mmol})$ in anhydrous THF $(300 \mathrm{~mL})$ was added potassium tert-butoxide $(45.5 \mathrm{~g}, 405 \mathrm{mmol})$ at $0{ }^{\circ} \mathrm{C}$ under $\mathrm{N}_{2}$ atmosphere. The reaction mixture was stirred at $0{ }^{\circ} \mathrm{C}$ for $20 \mathrm{~min}$ and then at room temperature for $1 \mathrm{~h}$. After the mixture was allowed to cool to $0{ }^{\circ} \mathrm{C}$, a solution of $\mathbf{1 1}(29.6 \mathrm{~g}, 129 \mathrm{mmol})$ in anhydrous THF $(50 \mathrm{~mL})$ was added and the mixture was stirred at room temperature overnight. To this mixture was carefully added water $(200 \mathrm{~mL})$ and the mixture was extracted with ethyl acetate $(800 \mathrm{~mL})$, dried, filtered, and concentrated under reduced pressure to give a yellow syrup. This syrup was purified by silica gel column chromatography (ethyl acetate/petroleum ether 1:20) to give $\mathbf{1 2}$ with the contamination of triphenylphosphine oxide, which was used for next reaction without further purification; ${ }^{1} \mathrm{H}$ NMR (400 $\mathrm{MHz}, \mathrm{CDCl}_{3}$ ): $\delta 5.91$ (ddd, $J=6.0,10.4,16.8 \mathrm{~Hz}, 1 \mathrm{H}), 5.41$ (d, $J=17.2 \mathrm{~Hz}, 1 \mathrm{H}), 5.21(\mathrm{~d}$, $J=10.4 \mathrm{~Hz}, 1 \mathrm{H}), 4.36(\mathrm{td}, J=1.2,6.4 \mathrm{~Hz}, 1 \mathrm{H}), 4.07-4.15(\mathrm{~m}, 2 \mathrm{H})$, 3.93-3.96 (m, 1H), $3.70(\mathrm{t}, J=7.2 \mathrm{~Hz}, 1 \mathrm{H}), 1.41(\mathrm{~s}, 6 \mathrm{H}), 1.40(\mathrm{~s}, 3 \mathrm{H})$, $1.34(\mathrm{~s}, 3 \mathrm{H}) ;{ }^{13} \mathrm{C}$ NMR $\left(100 \mathrm{MHz}, \mathrm{CDCl}_{3}\right): \delta 136.3,117.5,110.0,109.8$, 81.5, 80.8, 67.4, 27.3, 27.3, 27.0, 25.6; ESI-HRMS calcd for $\mathrm{C}_{12} \mathrm{H}_{20} \mathrm{O}_{4}$ $\left([\mathrm{M}+\mathrm{Na}]^{+}\right)$251.1253, found 251.1237.

4.1.2. (S)-1-((4S,5S)-2,2-Dimethyl-5-vinyl-1,3-dioxolan-4-yl)ethane1,2-diol (13). Compound 12 was added to $75 \%$ acetic acid ( $200 \mathrm{~mL})$ and the mixture was stirred at room temperature overnight. The reaction mixture was azeotroped with toluene under reduced pressure below $30^{\circ} \mathrm{C}$ to give a yellow syrup. This syrup was purified 
by silica gel column chromatography (ethyl acetate/petroleum ether $1: 1)$ to give $\mathbf{1 3}\left(18.6 \mathrm{~g}, 77 \%\right.$ from 11) as a colorless oil; $[\alpha]_{\mathrm{D}}^{30}$ -65.4 (c 0.7, $\left.\mathrm{CHCl}_{3}\right)$; IR $(\nu)$ : 3308, 2986, 1372, 1225, 1168, $1062 \mathrm{~cm}^{-1}$; ${ }^{1} \mathrm{H}$ NMR (400 MHz, $\mathrm{CDCl}_{3}$ ): $\delta 5.89$ (ddd, $J=6.8,10.0,16.8 \mathrm{~Hz}, 1 \mathrm{H}$ ), $5.43(\mathrm{~d}, J=16.8 \mathrm{~Hz}, 1 \mathrm{H}), 5.26(\mathrm{~d}, J=10.4 \mathrm{~Hz}, 1 \mathrm{H}), 4.41(\mathrm{td}, J=0.8$, $7.2 \mathrm{~Hz}, 1 \mathrm{H}), 3.85-3.88(\mathrm{~m}, 1 \mathrm{H}), 3.79(\mathrm{dd}, J=5.2,8.0 \mathrm{~Hz}, 1 \mathrm{H})$, 3.67-3.75 (m, 2H), 2.25 (br, 2H), 1.43 (s, 3H), $1.42(\mathrm{~s}, 3 \mathrm{H}) ;{ }^{13} \mathrm{C} \mathrm{NMR}$ $\left(100 \mathrm{MHz}, \mathrm{CDCl}_{3}\right): \delta 136.3,118.7,109.7,81.3,79.7,72.5,63.8,27.2$, 27.2; ESI-HRMS calcd for $\mathrm{C}_{9} \mathrm{H}_{16} \mathrm{O}_{4}\left([\mathrm{M}+\mathrm{Na}]^{+}\right)$211.0940, found 211.0917.

4.1.3. (4R,5S)-2,2-Dimethyl-4-((S)-oxiran-2-yl)-5-vinyl-1, 3dioxolane (9). To a stirred solution of 13 ( $4 \mathrm{~g}, 21.3 \mathrm{mmol}), \mathrm{Bu}_{2} \mathrm{SnO}$ (106 $\mathrm{mg}, 0.43 \mathrm{mmol})$, and triethylamine $(2.967 \mathrm{~mL}, 21.3 \mathrm{mmol})$ in dry $\mathrm{CH}_{2} \mathrm{Cl}_{2}, p$-toluenesulfonyl chloride $(4.07 \mathrm{~g}, 21.3 \mathrm{mmol})$ was added at $0{ }^{\circ} \mathrm{C}$ under $\mathrm{N}_{2}$ atmosphere. The resulting solution was stirred for $4 \mathrm{~h}$ at room temperature. Water was added and the reaction mixture was extracted with $\mathrm{CH}_{2} \mathrm{Cl}_{2}$. The organic extract was washed with brine, dried, and concentrated under reduced pressure to afford the crude tosylate $\mathbf{1 4}$ as a colorless oil, which was used for next step without any purification. To a stirred solution of 14 in $\mathrm{MeOH}(50 \mathrm{~mL})$, anhydrous $\mathrm{K}_{2} \mathrm{CO}_{3}(8.83 \mathrm{~g}$, $64.0 \mathrm{mmol})$ was added under $\mathrm{N}_{2}$ atmosphere. The resulting solution was stirred for $3 \mathrm{~h}$ at room temperature and filtered. The residue was washed with $\mathrm{CH}_{2} \mathrm{Cl}_{2}$, and the combined filtrates were concentrated under reduced pressure below $30^{\circ} \mathrm{C}$. The residue was redissolved in $\mathrm{CH}_{2} \mathrm{Cl}_{2}$, washed with brine, dried, and concentrated to give the crude product, which was purified by silica gel column chromatography (ethyl acetate/petroleum ether 1:40) to give $\mathbf{9}$ ( $2.82 \mathrm{~g}, 78 \%$ from 13) as a colorless oil; $[\alpha]_{\mathrm{D}}^{30} 25.1$ (c 1.3, $\left.\mathrm{CHCl}_{3}\right)$; IR $(\nu): 2957,2926,1653$, $1373,1259,1218,1176,1062,870 \mathrm{~cm}^{-1} ;{ }^{1} \mathrm{H} \operatorname{NMR}\left(400 \mathrm{MHz}, \mathrm{CDCl}_{3}\right)$ : $\delta 5.86$ (ddd, $J=6.8,10.4,17.2 \mathrm{~Hz}, 1 \mathrm{H}$ ), 5.42 (dd, $J=0.8,17.2 \mathrm{~Hz}, 1 \mathrm{H}$ ), 5.27 (dd, $J=0.8,10.4 \mathrm{~Hz}, 1 \mathrm{H}), 4.35(\mathrm{t}, J=7.2 \mathrm{~Hz}, 1 \mathrm{H}), 3.59$ (dd, $J=5.2$, $8.0 \mathrm{~Hz}, 1 \mathrm{H}), 3.06-3.09(\mathrm{~m}, 1 \mathrm{H}), 2.81(\mathrm{dd}, J=4.4,5.2 \mathrm{~Hz}, 1 \mathrm{H}), 2.69(\mathrm{dd}$, $J=2.8,4.8 \mathrm{~Hz}, 1 \mathrm{H}), 1.43(\mathrm{~s}, 3 \mathrm{H}), 1.42(\mathrm{~s}, 3 \mathrm{H}) ;{ }^{13} \mathrm{C}$ NMR $(100 \mathrm{MHz}$, $\left.\mathrm{CDCl}_{3}\right): \delta 135.3,119.2,110.1,80.6,80.4,51.5,45.0,27.2,27.0$; ESIHRMS calcd for $\mathrm{C}_{9} \mathrm{H}_{14} \mathrm{O}_{3}\left([\mathrm{M}+\mathrm{Na}]^{+}\right)$193.0835, found 193.0829 .

4.1.4. (S,E)-5-((4S,5R)-2,2-Dimethyl-5-((S)-oxiran-2-yl)-1,3-dioxolan-4-yl)pent-4-en-2-ol (8). To a stirred solution of $\mathbf{9}(1.17 \mathrm{~g}$, $6.9 \mathrm{mmol})$ and $10(885 \mathrm{mg}, 10.3 \mathrm{mmol})$ in dry $\mathrm{CH}_{2} \mathrm{Cl}_{2}(2 \mathrm{~mL}), 2 \% \mathrm{~mol}$ of Grubbs second generation catalyst was added under $\mathrm{N}_{2}$ atmosphere. The reaction mixture was stirred for $24 \mathrm{~h}$ under reflux. The reaction mixture was concentrated under reduced pressure. The residue was purified by silica gel column chromatography (ethyl acetate/petroleum ether $1: 6)$ to give $\mathbf{8}(1.33 \mathrm{~g}, 85 \%)$ as a colorless oil; $[\alpha]_{\mathrm{D}}^{30} 64.7$ ( c 1.3, $\mathrm{CHCl}_{3}$ ); IR $(\nu)$ : 3433, 2985, 2921, 1597, 1454, 1376, 1219, 1160, 1112, $1058 \mathrm{~cm}^{-1} ;{ }^{1} \mathrm{H}$ NMR (400 MHz, $\left.\mathrm{CDCl}_{3}\right)$ : $\delta$ 5.85-5.93 (m, 1H), 5.56-5.62 (m, 1H), $4.37(\mathrm{t}, J=8.0 \mathrm{~Hz}, 1 \mathrm{H})$, $3.83-3.91(\mathrm{~m}, 1 \mathrm{H}), 3.51(\mathrm{dd}, J=5.6,8.4 \mathrm{~Hz}, 1 \mathrm{H}), 3.06(\mathrm{ddd}, J=2.4,3.6$, $6.4 \mathrm{~Hz}, 1 \mathrm{H}), 2.82(\mathrm{dd}, J=4.0,4.8 \mathrm{~Hz}, 1 \mathrm{H}), 2.70(\mathrm{dd}, J=2.4,4.8 \mathrm{~Hz}, 1 \mathrm{H})$, 2.23-2.25 (m, 2H), 1.67 (br, 1H), $1.44(\mathrm{~s}, 3 \mathrm{H}), 1.43(\mathrm{~s}, 3 \mathrm{H}), 1.21(\mathrm{~d}$, $J=6.0 \mathrm{~Hz}, 3 \mathrm{H}) ;{ }^{13} \mathrm{C}$ NMR $\left(100 \mathrm{MHz}, \mathrm{CDCl}_{3}\right): \delta$ 132.8, 129.8, 109.8, 80.3, 80.2, 67.2, 51.3, 44.9, 42.2, 27.1, 26.8, 23.0; ESI-HRMS calcd for $\mathrm{C}_{12} \mathrm{H}_{20} \mathrm{O}_{4}\left([\mathrm{M}+\mathrm{Na}]^{+}\right)$251.1253, found 251.1264.

4.1.5. (S,E)-5-((4S,5S)-5-((S)-1-Hydroxy-4-(trimethylsilyl)but-3-yn1-yl)-2, 2-dimethyl-1,3-dioxolan-4-yl)pent-4-en-2-ol (15). To a stirred solution of trimethylsilylacetylene $(1.24 \mathrm{~mL}, 8.76 \mathrm{mmol})$ in anhydrous THF $(20 \mathrm{~mL})$, was added $n$-BuLi $(1.6 \mathrm{M}$ in hexanes, $5.34 \mathrm{~mL}, 8.54 \mathrm{mmol}$ ) dropwise at $-78^{\circ} \mathrm{C}$ under $\mathrm{N}_{2}$ atmosphere. The resulting solution was slowly warmed to $0{ }^{\circ} \mathrm{C}$ and stirred at this temperature for $1 \mathrm{~h}$. The reaction mixture was then cooled to $-78{ }^{\circ} \mathrm{C}$ and $\mathrm{BF}_{3} \cdot \mathrm{Et}_{2} \mathrm{O}(0.60 \mathrm{~mL}, 4.82 \mathrm{mmol})$ was added dropwise. After $20 \mathrm{~min}$ at $-78{ }^{\circ} \mathrm{C}$, a solution of $\mathbf{8}(500 \mathrm{mg}, 2.19 \mathrm{mmol})$ in THF $(20 \mathrm{~mL})$ was added via cannula. After $1 \mathrm{~h}$ of stirring at this temperature, the reaction mixture was hydrolyzed by adding brine $(30 \mathrm{~mL})$. The aqueous phase was extracted with ethyl acetate $(3 \times 50 \mathrm{~mL})$ and the combined organic layers were dried over anhydrous $\mathrm{Na}_{2} \mathrm{SO}_{4}$, filtered, and concentrated under reduced pressure. The residue was purified by silica gel column chromatography (ethyl acetate/petroleum ether 1:4) to give 15 (554 mg, 78\%) as a colorless oil; $[\alpha]_{\mathrm{D}}^{30} 50\left(c 0.6, \mathrm{CHCl}_{3}\right)$; IR $(\nu)$ : $3415,2985,2964,2932$, 2901, 2177, 1372, 1250, $1064 \mathrm{~cm}^{-1}$; ${ }^{1} \mathrm{H}$ NMR (400 $\left.\mathrm{MHz}, \mathrm{CDCl}_{3}\right)$ : $\delta 5.84-5.92(\mathrm{~m}, 1 \mathrm{H}), 5.63(\mathrm{dd}, J=8.0,15.6 \mathrm{~Hz}, 1 \mathrm{H}), 4.43(\mathrm{t}, J=8.0 \mathrm{~Hz}$, $1 \mathrm{H}), 3.85-3.95(\mathrm{~m}, 2 \mathrm{H}), 3.80(\mathrm{dd}, J=5.6,8.0 \mathrm{~Hz}, 1 \mathrm{H}), 2.51(\mathrm{dd}, J=1.6$, $6.8 \mathrm{~Hz}, 2 \mathrm{H}), 2.22-2.26(\mathrm{~m}, 2 \mathrm{H}), 1.95(\mathrm{br}, 2 \mathrm{H}), 1.42(\mathrm{~s}, 3 \mathrm{H}), 1.41(\mathrm{~s}, 3 \mathrm{H})$, $1.20(\mathrm{~d}, J=6.0 \mathrm{~Hz}, 3 \mathrm{H}), 0.156(\mathrm{~s}, 9 \mathrm{H}) ;{ }^{13} \mathrm{C}$ NMR (100 MHz, $\left.\mathrm{CDCl}_{3}\right)$ : $\delta$ 132.1, 131.0, 108.9, 102.4, 87.6, 81.7, 78.7, 69.8, 67.0, 42.0, 27.0, 26.9, 25.0, 22.8, 0.0; ESI-HRMS calcd for $\mathrm{C}_{17} \mathrm{H}_{30} \mathrm{O}_{4} \mathrm{Si}\left([\mathrm{M}+\mathrm{Na}]^{+}\right)$349.1805, found 349.1815 .

4.1.6. (S,E)-5-((4S,5S)-5-((S)-1-Hydroxybut-3-ynyl)-2,2-dimethyl1,3-dioxolan-4-yl)pent-4-en-2-ol (16). To a solution of 15 (397 mg, $1.22 \mathrm{mmol})$ in $\mathrm{MeOH}(10 \mathrm{~mL})$, was added $\mathrm{K}_{2} \mathrm{CO}_{3}(252 \mathrm{mg}$, $1.83 \mathrm{mmol}$ ). The mixture was stirred for $12 \mathrm{~h}$ at room temperature before water $(5 \mathrm{~mL})$ was added. The mixture was extracted with $\mathrm{CH}_{2} \mathrm{Cl}_{2}$. The combined organic layers were dried over $\mathrm{Na}_{2} \mathrm{SO}_{4}$, filtered, and concentrated. The residue was purified by silica gel column chromatography (ethyl acetate/petroleum ether $1: 3$ ) to give 16 (268 mg, 86\%) as a colorless oil; $[\alpha]_{D}^{30} 45\left(c 0.6, \mathrm{CHCl}_{3}\right)$; IR $(\nu)$ : 3301, 2964, 2924, 2856, 2119, 1670, 1374, $1062 \mathrm{~cm}^{-1}$; ${ }^{1} \mathrm{H}$ NMR $\left(400 \mathrm{MHz}, \mathrm{CDCl}_{3}\right): \delta 5.83-5.92(\mathrm{~m}, 1 \mathrm{H}), 5.65(\mathrm{dd}, J=7.6,15.6 \mathrm{~Hz}, 1 \mathrm{H})$, $4.43(\mathrm{t}, J=7.6 \mathrm{~Hz}, 1 \mathrm{H}), 3.92-3.96(\mathrm{~m}, 1 \mathrm{H}), 3.86-3.90(\mathrm{~m}, 1 \mathrm{H}), 3.81$ $(\mathrm{dd}, J=5.2,7.6 \mathrm{~Hz}, 1 \mathrm{H}), 2.48-2.51(\mathrm{~m}, 2 \mathrm{H}), 2.18-2.31(\mathrm{~m}, 2 \mathrm{H}), 2.07(\mathrm{t}$, $J=2.8 \mathrm{~Hz}, 1 \mathrm{H}), 1.69(\mathrm{br}, 2 \mathrm{H}), 1.42(\mathrm{~s}, 3 \mathrm{H}), 1.41(\mathrm{~s}, 3 \mathrm{H}), 1.20(\mathrm{~d}, J=6.4 \mathrm{~Hz}$, $3 \mathrm{H}) ;{ }^{13} \mathrm{C}$ NMR (100 MHz, $\mathrm{CDCl}_{3}$ ): $\delta$ 132.0, 131.7, 109.4, 81.9, 80.2, 79.1, 71.6, 70.3, 67.4, 42.3, 27.4, 27.3, 23.9, 23.2; ESI-HRMS calcd for $\mathrm{C}_{14} \mathrm{H}_{22} \mathrm{O}_{4}\left([\mathrm{M}+\mathrm{Na}]^{+}\right) 277.1444$, found 277.1412 .

4.1.7. (S,E)-5-((4S,5S)-5-((S)-4-Bromo-1-hydroxybut-3-ynyl)-2,2-dimethyl-1,3-dioxolan-4-yl)pent-4-en-2-ol (17). To a solution of $\mathbf{1 6}$ $(218 \mathrm{mg}, 0.86 \mathrm{mmol})$ in acetone $(25 \mathrm{~mL})$, was added silver nitrate (44 mg, $0.26 \mathrm{mmol}$ ) and $\mathrm{N}$-bromosuccinimide (183 $\mathrm{mg}, 1.03 \mathrm{mmol}$ ). The reaction mixture was stirred for $1 \mathrm{~h}$, poured into saturated $\mathrm{NaHCO}_{3}(5 \mathrm{~mL})$ and saturated sodium thiosulfate $(5 \mathrm{~mL})$, and extracted with $\mathrm{CH}_{2} \mathrm{Cl}_{2}$. The combined extracts were dried over $\mathrm{Na}_{2} \mathrm{SO}_{4}$ and concentrated under reduced pressure. The residue was purified by silica gel column chromatography (ethyl acetate/petroleum ether $1: 3)$ to give $\mathbf{1 7}(278 \mathrm{mg}, 97 \%)$ as a colorless oil; $[\alpha]_{D}^{30}$ 73.8 ( $c$ 0.9, $\mathrm{CHCl}_{3}$ ); IR ( $\nu$ ): 3374, 2960, 2921, 2853, 2309, 1603, 1376, $1123,1059 \mathrm{~cm}^{-1}$; ${ }^{1} \mathrm{H}$ NMR $\left(400 \mathrm{MHz}, \mathrm{CDCl}_{3}\right): \delta 5.84-5.91(\mathrm{~m}, 1 \mathrm{H})$, $5.62(\mathrm{dd}, J=8.0,15.6 \mathrm{~Hz}, 1 \mathrm{H}), 4.41(\mathrm{t}, J=8.0 \mathrm{~Hz}, 1 \mathrm{H}), 3.85-3.94(\mathrm{~m}$, $2 \mathrm{H}$ ), 3.77 (dd, $J=5.6,8.0 \mathrm{~Hz}, 1 \mathrm{H}), 2.50(\mathrm{~d}, J=6.4 \mathrm{~Hz}, 2 \mathrm{H}), 2.22-2.26$ $(\mathrm{m}, 2 \mathrm{H}), 2.10(\mathrm{br}, 2 \mathrm{H}), 1.42(\mathrm{~s}, 3 \mathrm{H}), 1.40(\mathrm{~s}, 3 \mathrm{H}), 1.20(\mathrm{~d}, J=6.4 \mathrm{~Hz}, 3 \mathrm{H})$; ${ }^{13} \mathrm{C}$ NMR $\left(100 \mathrm{MHz}, \mathrm{CDCl}_{3}\right): \delta$ 132.4, 131.5, 109.4, 81.9, 79.1, 76.3, 70.2, 67.5, 42.2, 41.3, 27.4, 27.3, 25.1, 23.2; ESI-HRMS calcd for $\mathrm{C}_{14} \mathrm{H}_{21} \mathrm{BrO}_{4}\left([\mathrm{M}+\mathrm{Na}]^{+}\right)$355.0515, found 355.0514.

4.1.8. (S,E)-5-((4S,5S)-5-((S,E)-1-Hydroxy-4-(tributylstannyl)but-3enyl)-2,2-dimethyl-1, 3-dioxolan-4-yl)pent-4-en-2-ol (6). To a solution of the alkynyl bromide 17 (256 $\mathrm{mg}, 0.77 \mathrm{mmol})$ in anhydrous THF $(20 \mathrm{~mL})$, was added $\mathrm{Cl}_{2} \mathrm{Pd}\left(\mathrm{PPh}_{3}\right)_{2}(11 \mathrm{mg}, 0.0154 \mathrm{mmol})$ and tributyltin hydride $(0.42 \mathrm{~mL}, 1.54 \mathrm{mmol})$. The reaction mixture was stirred for $30 \mathrm{~min}$, and concentrated under reduced pressure. The residue was purified by silica gel column chromatography (ethyl acetate/petroleum ether 1:6) to give $\mathbf{6}(357 \mathrm{mg}, 85 \%)$ as a colorless oil; $[\alpha]_{\mathrm{D}}^{30} 28.8\left(c 0.8, \mathrm{CHCl}_{3}\right)$; IR $(\nu)$ : 3411, 2957, 2926, 2870, 2850, 1673, 1597, 1457, 1376, 1244, 1165, $1056 \mathrm{~cm}^{-1}$; ${ }^{1} \mathrm{H}$ NMR $(400 \mathrm{MHz}$, $\left.\mathrm{CDCl}_{3}\right): \delta 5.81-6.17(\mathrm{~m}, 3 \mathrm{H}), 5.60(\mathrm{dd}, J=8.0,15.6 \mathrm{~Hz}, 1 \mathrm{H}), 4.44(\mathrm{t}$, $J=8.0 \mathrm{~Hz}, 1 \mathrm{H}), 3.80-3.93(\mathrm{~m}, 2 \mathrm{H}), 3.70(\mathrm{dd}, J=4.8,8.0 \mathrm{~Hz}, 1 \mathrm{H})$, 2.16-2.51 (m, 5H), 2.06-2.10 (m, 1H), 1.46-1.71 (m, 6H), $1.42(\mathrm{~s}$, 
$3 \mathrm{H}), 1.41$ (s, 3H), $1.25-1.36(\mathrm{~m}, 6 \mathrm{H}), 1.20(\mathrm{~d}, J=6.4 \mathrm{~Hz}, 3 \mathrm{H}), 0.79-0.96$ $(\mathrm{m}, 15 \mathrm{H}) ;{ }^{13} \mathrm{C}$ NMR $\left(100 \mathrm{MHz}, \mathrm{CDCl}_{3}\right): \delta$ 144.4, 133.5, 132.0, 109.1, 82.8, 78.7, 70.7, 67.4, 42.4, 41.9, 29.5, 27.6, 27.4, 27.3, 23.2, 14.1, 9.8; ESI-HRMS calcd for $\mathrm{C}_{26} \mathrm{H}_{50} \mathrm{O}_{4} \mathrm{Sn}\left([\mathrm{M}+\mathrm{Na}]^{+}\right)$569.2628, found 569.2628 .

4.1.9. 5-((S,E)-4-Hydroxy-4-((4S,5S)-5-((S,E)-4-hydroxypent-1-en-1yl)-2,2-dimethyl-1, 3-dioxolan-4-yl)but-1-en-1-yl)-7-methoxy-2,2dimethyl-4H-benzo[d][1,3]dioxin-4-one (5). To a flame-dried flask containing triflate $7(107 \mathrm{mg}, 0.301 \mathrm{mmol}), \mathrm{Pd}(\mathrm{dba})_{2}(106 \mathrm{mg}$, $0.184 \mathrm{mmol}), \mathrm{AsPh}_{3}(56 \mathrm{mg}, 0.183 \mathrm{mmol}$ ), and $\mathrm{LiCl}(29 \mathrm{mg}$, $0.684 \mathrm{mmol}$ ), was added anhydrous DMF $3 \mathrm{~mL}$ under $\mathrm{N}_{2}$ atmosphere. The resulting mixture was stirred at room temperature for $30 \mathrm{~min}$ before addition of vinyl stannane $\mathbf{6}(126 \mathrm{mg}, 0.231 \mathrm{mmol})$. The reaction was heated to $60{ }^{\circ} \mathrm{C}$ for $2 \mathrm{~h}$, cooled to room temperature, and then concentrated under reduced pressure. The residue was purified by silica gel column chromatography (ethyl acetate/ petroleum ether $1: 1)$ to give $\mathbf{5}(86 \mathrm{mg}, 81 \%)$ as a colorless oil; $[\alpha]_{D}^{30}$ 16.7 (c 0.6, $\mathrm{CHCl}_{3}$ ); IR ( $\nu$ ): 3372, 2923, 1726, 1659, 1603, 1460, 1429, $1379 \mathrm{~cm}^{-1}$; ${ }^{1} \mathrm{H}$ NMR $\left(400 \mathrm{MHz}, \mathrm{CDCl}_{3}\right): \delta 7.37(\mathrm{~d}, J=16.0 \mathrm{~Hz}, 1 \mathrm{H})$, 6.69 (d, $J=2.4 \mathrm{~Hz}, 1 \mathrm{H}), 6.34(\mathrm{~d}, J=2.4 \mathrm{~Hz}, 1 \mathrm{H}), 6.09-6.16(\mathrm{~m}, 1 \mathrm{H})$, 5.85-5.92 (m, 1H), 5.63 (dd, J=7.6, $15.2 \mathrm{~Hz}, 1 \mathrm{H}$ ), 4.47 (t, $J=7.6 \mathrm{~Hz}$, $1 \mathrm{H}), 3.83-3.93(\mathrm{~m}, 5 \mathrm{H}), 3.73(\mathrm{dd}, J=5.2,8.0 \mathrm{~Hz}, 1 \mathrm{H}), 2.34-2.56(\mathrm{~m}$, $3 \mathrm{H}), 2.10-2.28(\mathrm{~m}, 3 \mathrm{H}), 1.69(\mathrm{~s}, 6 \mathrm{H}), 1.41(\mathrm{~s}, 6 \mathrm{H}), 1.18(\mathrm{~d}, J=6.0 \mathrm{~Hz}$, $3 \mathrm{H}) ;{ }^{13} \mathrm{C}$ NMR $\left(100 \mathrm{MHz}, \mathrm{CDCl}_{3}\right): \delta 165.3,160.7,159.0,144.1,132.2$, 132.2, 131.7, 130.3, 109.1, 109.0, 105.5, 104.0, 100.7, 82.8, 79.1, 71.4, $67.5,56.0,42.3,37.0,27.4,27.3,25.9,23.2$; ESI-HRMS calcd for $\mathrm{C}_{25} \mathrm{H}_{34} \mathrm{O}_{8}\left([\mathrm{M}+\mathrm{Na}]^{+}\right)$485.2145, found 485.2130.

4.1.10. Cochliomycin A (1). To a stirred solution of 5 (57 mg, $0.123 \mathrm{mmol}$ ) in anhydrous DMF ( $10 \mathrm{~mL}$ ), was added sodium hydride ( $86 \mathrm{mg}, 2.15 \mathrm{mmol}, 60 \%$ oil dispersion) at $0{ }^{\circ} \mathrm{C}$ under $\mathrm{N}_{2}$ atmosphere. The mixture was stirred for $1 \mathrm{~h}$. To this mixture was added saturated aqueous $\mathrm{NH}_{4} \mathrm{Cl}$ and the mixture was extracted with ethyl acetate, dried, filtered, and concentrated under reduced pressure. This residue was purified by silica gel column chromatography (ethyl acetate/petroleum ether 1:3) to give 1 (23 $\mathrm{mg}, 46 \%$ ) as a white solid $\left(\operatorname{mp~} 68^{\circ} \mathrm{C}\right) ;[\alpha]_{\mathrm{D}}^{24} 10.5(c 0.6, \mathrm{MeOH}) ; \operatorname{IR}(\nu): 3405,2921$, 2372, 2307, 1629, $1378 \mathrm{~cm}^{-1}$; ${ }^{1} \mathrm{H}$ NMR (400 MHz, $\left.\mathrm{CDCl}_{3}\right): \delta 11.49$ (s, $1 \mathrm{H}), 7.17$ (dd, $J=2.0,15.2 \mathrm{~Hz}, 1 \mathrm{H}), 6.48(\mathrm{~d}, J=2.8 \mathrm{~Hz}, 1 \mathrm{H}), 6.40$ (d, $J=2.8 \mathrm{~Hz}, 1 \mathrm{H}$ ), 6.00 (ddd, $J=5.2,8.0,15.2 \mathrm{~Hz}, 1 \mathrm{H}$ ), 5.73 (ddd, $J=2.8$, $10.4,15.2 \mathrm{~Hz}, 1 \mathrm{H}), 5.53$ (dd, $J=8.8,15.2 \mathrm{~Hz}, 1 \mathrm{H}), 5.42-5.48(\mathrm{~m}, 1 \mathrm{H})$, $4.57(\mathrm{t}, J=8.4 \mathrm{~Hz}, 1 \mathrm{H}), 4.21$ (ddd, $J=2.0,4.8,12.0 \mathrm{~Hz}, 1 \mathrm{H}), 3.90$ (dd, $J=2.4,8.0 \mathrm{~Hz}, 1 \mathrm{H}), 3.82(\mathrm{~s}, 3 \mathrm{H}), 2.73-2.80(\mathrm{~m}, 1 \mathrm{H}), 2.18-2.55(\mathrm{~m}$, $4 \mathrm{H}), 1.45$ (d, $J=6.4 \mathrm{~Hz}, 3 \mathrm{H}), 1.44(\mathrm{~s}, 3 \mathrm{H}), 1.38(\mathrm{~s}, 3 \mathrm{H}) ;{ }^{13} \mathrm{C} \mathrm{NMR}$ $\left(100 \mathrm{MHz}, \mathrm{CDCl}_{3}\right): \delta 170.8,164.8,164.0,142.1,134.1,132.7,129.5$, 126.4, 108.5, 107.2, 104.4, 100.1, 81.4, 75.3, 70.6, 68.8, 55.4, 37.9, 36.0, 27.0, 27.0, 19.2; ESI-HRMS calcd for $\mathrm{C}_{22} \mathrm{H}_{28} \mathrm{O}_{7}\left([\mathrm{M}+\mathrm{Na}]^{+}\right)$427.1727, found 427.1728 .

\section{Acknowledgements}

This work was supported in partial by the National Basic Research Program of China (Grants 2012CB822101 and 2011CB936001), National Innovative Drug Foundation (2012ZX09502001), and NNSF of China (Projects 21372254 and
21202193). We thank Min Li and Yuan Li for MS measurements. We thank Jinshan Li for IR measurements. We also thank Dr. YiXian Li (CAS Key Laboratory of Molecular Recognition and Function, Institute of Chemistry, Chinese Academy of Sciences) for help in measuring the optical rotation of Cochliomycin A.

\section{Supplementary data}

Supplementary data related to this article can be found at http:// dx.doi.org/10.1016/j.tet.2014.03.001.

\section{References and notes}

1. (a) Winssinger, N.; Barluenga, S. Chem. Commun. 2007, 22-36; (b) Shier, W. T.; Shier, A. C.; Xie, W.; Mirocha, C. J. Toxicon 2001, 39, 1435-1438; (c) Isaka, M.; Yangchum, A.; Intamas, S.; Kocharin, K.; Jones, E. B. G.; Kongsaeree, P.; Prabpai, S. Tetrahedron 2009, 65, 4396-4403; (d) Dong, J.; Zhu, Y.; Song, H.; Li, R.; He, H.; Liu, H.; Huang, R.; Zhou, Y.; Wang, L.; Cao, Y.; Zhang, K. J. Chem. Ecol. 2007, 33 1115-1126; (e) Abid, E. S.; Ouanes, Z.; Hassen, W.; Baudrimont, I.; Creppy, E. Bacha, H. Toxicol. In Vitro 2004, 18, 467-474; (f) Chanmugam, P.; Feng, L.; Liou, S.; Jang, B. C.; Boudreau, M.; Yu, G.; Lee, J. H.; Kwon, H. J.; Beppu, T.; Yoshida, M.; Xia, Y.; Wilson, C. B.; Hwang, D. J. Biol. Chem. 1995, 270, 5418-5426; (g) Takehara, K.; Sato, S.; Kobayashi, T.; Maeda, T. Biochem. Biophys. Res. Commun. 1999, 257, 19-23.

2. Shao, C. L.; Wu, H. X.; Wang, C. Y.; Liu, Q. A.; Xu, Y.; Wei, M. Y.; Qian, P. Y.; Gu, Y. C.; Zheng, C. J.; She, Z. G.; Lin, Y. C. J. Nat. Prod. 2011, 74, 629-633.

3. Jana, N.; Nanda, S. Eur. J. Org. Chem. 2012, 23, 4313-4320.

4. (a) Zhang, C.; Liu, J.; Du, Y. Tetrahedron Lett. 2013, 54, 3278-3280; (b) Zhang, C. Liu, J.; Du, Y. Tetrahedron Lett. 2014, http://dx.doi.org/10.1016/j.tetlet.2013.12. 052; (c) Liu, J; Zhang, X.; Liu, Y.; Bi, J.; Du, Y. Sci. China Chem. 2013, 56, 928-932; (d) Liu, J.; Liu, Y.; Zhang, X.; Zhang, C.; Gao, Y.; Wang, L.; Du, Y. J. Org. Chem. 2012, 77, 9718-9723; (e) Cai, C.; Liu, J.; Du, Y.; Linhardt, R. J. J. Org. Chem. 2010 75, 5754-5756; (f) Du, Y.; Liu, J.; Linhardt, R. J. J. Org. Chem. 2006, 71, 1251-1253; (g) Du, Y.; Chen, Q.; Linhardt, R. J. J. Org. Chem. 2006, 71, 8446-8451.

5. Horton, D.; Varela, O. Carbohydr. Res. 1984, 134, 205-214.

6. Moon, H. R.; Choi, W. J.; Kim, H. O.; Jeong, L. S. Tetrahedron: Asymmetry 2002, 13 , 1189-1193.

7. Lewbart, M. L.; Schneider, J. J. J. Org. Chem. 1969, 34, 3505-3512.

8. (a) Martinelli, M. J.; Nayyar, N. K.; Moher, E. D.; Dhokte, U. P.; Pawlak, J. M.; Vaidyanathan, R. Org. Lett. 1999, 1, 447-450; (b) Martinelli, J.; Vaidyanathan, R. Pawlak, J. M.; Nayyar, N. K.; Dhokte, U. P.; Doecke, C. W.; Zollars, L. M. H.; Moher, E. D.; Khau, V. V.; Kosmrlj, B. J. Am. Chem. Soc. 2002, 124, 3578-3585; (c) Martinelli, M. J.; Vaidyanathan, R.; Khau, V. V. Tetrahedron Lett. 2000, 41 $3773-3776$.

9. Kamal, A.; Vangala, S. R. Tetrahedron 2011, 67, 1341-1347.

10. (a) Kumar, P.; Gupta, P.; Naidu, S. V. Chem.-Eur. J. 2006, 12, 1397-1402; (b) Rao, D. C.; Reddy, D. K.; Shekhar, V.; Venkateswarlu, Y. Tetrahedron Lett. 2013, 54, $828-829$.

11. (a) Scholl, M.; Ding, S.; Lee, C. W.; Grubbs, R. H. Org. Lett. 1999, 1, 953-956; (b) Chatterjee, A. K.; Choi, T. L.; Sanders, D. P.; Grubbs, R. H. J. Am. Chem. Soc. 2003, 125, 11360-11370; (c) Srinivas, B.; Sridhar, R.; Rama Rao, K. Tetrahedron 2010 66, 8527-8535; (d) Vamshikrishna, K.; Srihari, P. Tetrahedron 2012, 68, 1540-1546.

12. (a) Yamaguchi, M.; Hirao, I. Tetrahedron Lett. 1983, 24, 391-394; (b) Amans, D.; Bareille, L.; Bellosta, V.; Cossy, J. J. Org. Chem. 2009, 74, 7665-7674.

13. Hofmeister, H.; Annen, K.; Laurent, H.; Weichert, R. Angew. Chem., Int. Ed. Engl. 1984, 23, 727-729.

14. Zhang, H. X.; Guibe, F.; Balavoine, G. J. Org. Chem. 1990, 55, 1857-1867.

15. Srihari, P.; Mahankali, B.; Rajendraprasad, K. Tetrahedron Lett. 2012, 53, 56-58. 16. Farina, V.; Krishnan, B. J. Am. Chem. Soc. 1991, 113, 9585-9595.

17. Inanaga, J.; Hirata, K.; Saeki, H.; Katsuki, T.; Yamaguchi, M. Bull. Chem. Soc. Jpn. 1979, 52, 1989-1993.

18. (a) Corey, E. J.; Nicolaou, K. C. J. Am. Chem. Soc. 1974, 96, 5614-5616; (b) Buszek, K. R.; Sato, N.; Jeong, Y. J. Am. Chem. Soc. 1994, 116, 5511-5512.

19. (a) Bhattacharjee, A.: De Brabander, J. K. Tetrahedron Lett. 2000, 41, 8069-8073; (b) Nicolaou, K. C.; Kim, D. W.; Baati, R. Angew. Chem., Int. Ed. 2002, 41, 3701-3704; (c) Rountree, J. S. S.; Murphy, P. V. Org. Lett. 2009, 11, 871-874. 